\title{
Laboratory diagnosis of galactosemia: a technical standard and guideline of the American College of Medical Genetics and Genomics (ACMG)
}

\author{
Marzia Pasquali, $\mathrm{PhD}^{1}$, Chunli Yu, MD² and Bradford Coffee, $\mathrm{PhD}^{3}$; \\ on behalf of the ACMG Laboratory Quality Assurance Committee
}

\begin{abstract}
Disclaimer: These ACMG Standards and Guidelines are developed primarily as an educational resource for clinical laboratory geneticists to help them provide quality clinical laboratory genetic services. Adherence to these Standards and Guidelines is voluntary and does not necessarily assure a successful medical outcome. These Standards and Guidelines should not be considered inclusive of all proper procedures and tests or exclusive of other procedures and tests that are reasonably directed to obtaining the same results. In determining the propriety of any specific procedure or test, the clinical laboratory geneticist should apply his or her own professional judgment to the specific circumstances presented by the individual patient or specimen. Clinical laboratory geneticists are encouraged to document in the patient's record the rationale for the use of a particular procedure or test, whether or not it is in conformance with these Standards and Guidelines. They also are advised to take notice of the date any particular guideline was adopted, and to consider other relevant medical and scientific information that becomes available after that date. It also would be prudent to consider whether intellectual property interests may restrict the performance of certain tests and other procedures.
\end{abstract}

Galactosemias are inherited disorders of galactose metabolism due to deficiency in one of the three enzymes involved in the Leloir pathway: galactose-1-phosphate uridyltransferase, galactokinase, and uridine diphosphate (UDP)-galactose-4'-epimerase. Galactose-1-phosphate uridyltransferase deficiency, or classic galactosemia, is the most frequent and the most severe of the three enzyme deficiencies; it is characterized by failure to thrive, liver failure, susceptibility to sepsis, and death, if untreated. Newborn screening for classic galactosemia has been implemented in all of the United States, while screening for galactokinase deficiency and UDP-galactose- 4 '-epimerase deficiency is not universal. Early identification and treatment of galactosemia leads to improved outcome. This document reviews the laboratory methods and best practices for the diagnosis of galactosemia.

Genet Med advance online publication 26 October 2017

Key Words: enzyme assay; galactokinase; galactose-1-phosphate uridyltransferase; galactosemia; UDP-galactose-4'-epimerase

\section{METHODS}

This laboratory technical standard and guideline was informed by a review of the literature, including any current guidelines, and expert opinion. Resources consulted included PubMed (search terms: galactosemia, galactose-1-phosphate uridyltransferase, galactokinase, galactose epimerase, galactose-1-phosphate, Duarte variant), the American College of Medical Genetics and Genomics (ACMG) Technical Standards and Guidelines for Clinical Genetics Laboratories, Clinical and Laboratory Standards Institute guidelines, CLIA regulations, and the Centers for Disease Control and Prevention's Morbidity and Mortality Weekly Report on Good Laboratory Practices for Biochemical Genetics Testing and Newborn Screening for Inherited Metabolic Disorders. When the literature provided conflicting evidence about a topic or when there was insufficient evidence, the authors used expert opinion to inform the recommendations. Expert opinion included the coauthors of the document, as well as any experts consulted outside the workgroup and acknowledged in this document. Any conflicts of interest for workgroup members or consultants are listed. The ACMG Laboratory Quality Assurance Committee (Lab QA) reviewed the document providing further input on the content, and a final draft was delivered to the ACMG Board of Directors for review and approval to send out for member comment. The final draft of the document was uploaded to the ACMG website and an e-mail link was sent to ACMG members inviting all to provide comment. All members' comments were assessed by

${ }^{1}$ University of Utah School of Medicine and ARUP Laboratories, Salt Lake City, Utah, USA; ${ }^{2}$ Mount Sinai Genetic Testing Laboratory, Icahn School of Medicine at Mount Sinai, New York, New York, USA; ${ }^{3}$ Myriad Genetics, Salt Lake City, Utah, USA. Correspondence: Marzia Pasquali (pasquam@aruplab.com)

The Board of Directors of the American College of Medical Genetics and Genomics approved this guideline on 22 May 2017.

Submitted 30 August 2017; accepted 30 August 2017; advance online publication 26 October 2017. doi:10.1038/gim.2017.172 


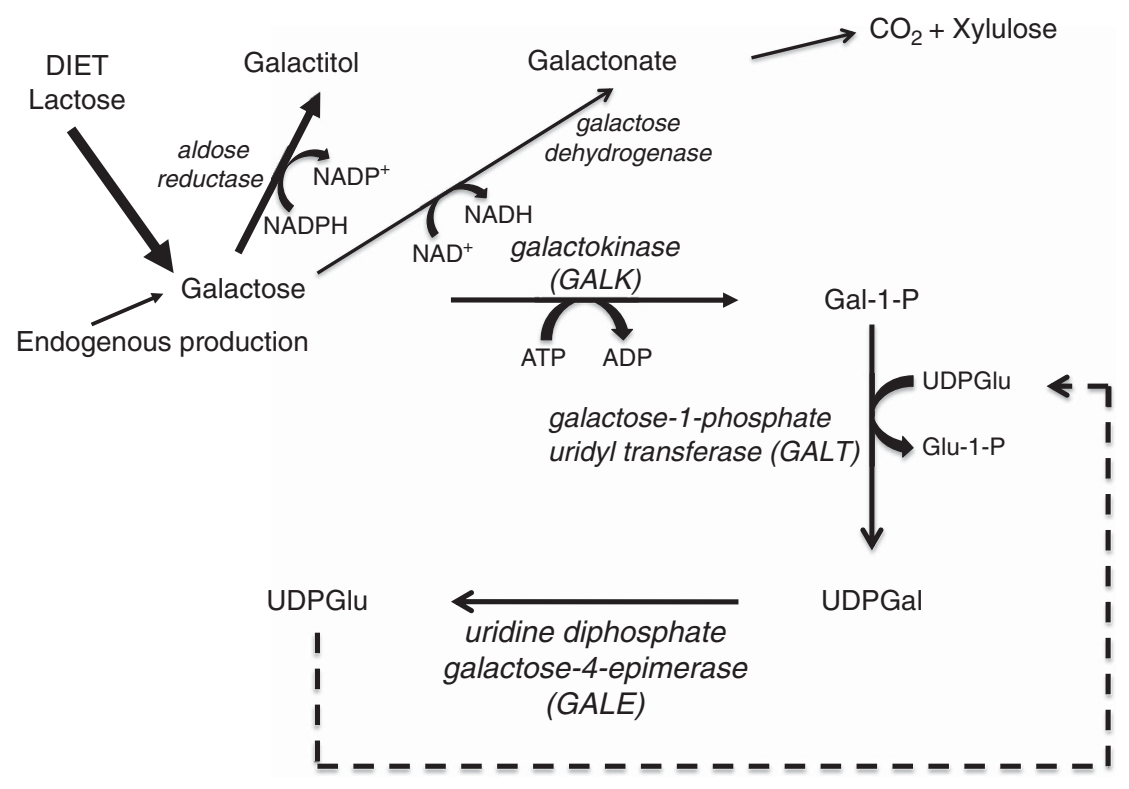

Figure 1 Metabolism of galactose. Galactose, deriving from diet and, in minor part, from endogenous synthesis, is phosphorylated to galactose-1phosphate (Gal-1-P) by galactokinase, the first enzyme in the Leloir pathway. Uridine diphosphate (UDP)-galactose is produced from Gal-1-P and UDPglucose through the enzyme galactose-1-phosphate uridyltransferase. UDP-galactose is then converted to UDP-glucose by the enzyme uridine diphosphate galactose-4-epimerase. UDP-glucose is recycled back into the pathway.

the authors and additional evidence was included; our recommendations were amended as deemed appropriate. Member comments and author responses were reviewed by a representative of the Lab QA committee and the ACMG Board. The final document was approved by the ACMG Board of Directors.

\section{OVERVIEW}

Galactosemia encompasses three distinct enzymatic deficiencies in the Leloir pathway of galactose metabolism (Figure 1). This pathway plays a critical role in converting D-galactose to glucose-1-phoshate for entry into glycolysis, as well as supplying uridine diphosphate galactose (UDP-galactose) for the galactosylation of carbohydrates and lipids. Classic galactosemia (MIM 230400) is caused by deficient galactose1-phosphate uridyltransferase (GALT) activity, leading to elevated galactose-1-phosphate (Gal-1-P) and other galactose metabolites in blood and urine. A variant form of galactosemia, referred to as Duarte variant or DG galactosemia, is characterized by approximately $25 \%$ of normal GALT activity and may be clinically benign. ${ }^{1}$ Galactokinase (GALK) deficiency and UDP-galactose-4'-epimerase (GALE) deficiency are clinically and biochemically distinct from classic galactosemia. For each of these conditions, the diagnosis is based on the demonstration of severely reduced or absent enzyme activity in red blood cells (RBCs). DNA analysis of the GALT, GALK1, or GALE genes is often performed as part of the diagnostic evaluation.

Because galactosemia is included in all newborn screening programs in the United States and many other countries worldwide, the majority of diagnostic testing originates from this referral source. Newborn screening for classic galactosemia identifies patients with decreased GALT activity in dried blood spots (DBS) and, for some states, elevated galactose sugars. States including galactose sugars as part of their screening also identify cases of GALK and GALE deficiencies, while those relying only on galactose sugars alone may miss cases of GALT deficiency in patients on a galactose-limited diet. Galactosemia testing may also be initiated by a positive family history, or by nonspecific features of the disease in an older patient. Although diagnostic testing is relatively straightforward, significant variability can result from preanalytic and analytic influences if not properly controlled. These guidelines were developed to delineate best practices for enzyme and metabolite testing for galactosemia and to characterize variables that can influence test performance and results interpretation.

\section{BACKGROUND}

Biochemical and molecular characteristics of galactosemetabolizing enzymes

Galactose-1-phosphate uridyltransferase

Galactose-1-phosphate uridyltransferase (GALT; EC 2.7.7.12), the second enzyme of the Leloir pathway, is a member of the histidine triad superfamily and is a homodimer composed of two $43-\mathrm{kDa}$ subunits. ${ }^{2}$ This enzyme catalyzes the interconversion of Gal-1-P to glucose-1-phosphate, with the concomitant interconversion of the co-substrate UDP-glucose to UDPgalactose. The reaction follows a "ping-pong" mechanism whereby histidine at the active site reacts with UDP-glucose loading the enzyme with uridine $5^{\prime}$-monophosphate (UMP); 
UMP then reacts with galactose-1-phosphate, forming the final product, UDP-galactose. ${ }^{3}$

The GALT gene encompasses 11 exons spanning $4.3 \mathrm{~kb}$ of chromosome 9p13. An open-access database has curated more than 250 pathogenic variants including nonsense, missense, frameshift, splice site variants, and large deletions. ${ }^{4}$ The vast majority $(\sim 85 \%)$ are classified as causing profound enzyme impairment consistent with classic galactosemia; of these, $61 \%$ are missense variants. Among patients of European descent, c.563A $>$ G (p.Q188R) is the most common variant and accounts for $64 \%$ of all pathogenic variants seen. ${ }^{5}$ The c.404C $>\mathrm{T}$ (p.S135L) variant is associated with an attenuated phenotype, and is found almost exclusively in patients of African origin. ${ }^{6}$ Other common pathogenic variants include: c.855G $>\mathrm{T}$ (p.K285N), c.626A $>\mathrm{G}$ (p. Y209C), c.413C > T (p.T138M), c.584T > C (p.L195P) and IVS2-2A $>$ G. ${ }^{5}$ A deletion of approximately $5.5 \mathrm{~kb}$ is common among Ashkenazi Jewish individuals. ${ }^{7,8}$ All of these variants are associated with near or complete loss of enzyme activity, although for p.S135L the deficiency may be confined to RBCs. ${ }^{6,9}$

Duarte variant (DG) galactosemia results from compound heterozygosity for a profound GALT pathogenic variant $(\mathrm{G})$ and a second allele known as Duarte 2 (D2). D2 occurs in an estimated $11 \%$ of European American populations, and was originally characterized by an abnormal banding pattern on gel electrophoresis or isoelectric focusing. It is now known to encompass a haplotype of four variants including two intronic changes, a 4-bp deletion in the promoter of the gene, and c.940A > G (p.N314D). The D2 allele reduces GALT activity by approximately $50 \%$, such that when present in trans with a classic galactosemia allele the net result is $\sim 25 \%$ of normal activity. Classic galactosemia pathogenic variants can occur in cis with D2 resulting in complete loss of activity on that allele. ${ }^{10}$ Current evidence supports the hypothesis that the 4-bp deletion is responsible for the reduction in enzyme activity. ${ }^{11,12}$ A second variant, Duarte 1 (D1, also known as the Los Angeles, or LA, variant), shares the D2 electrophoretic banding pattern but is not associated with reduced GALT activity. ${ }^{13}$ The D1 allele contains c.940A > G (p.N314D), a synonymous nucleotide change in exon 7, c.652C $>\mathrm{T}$ (p.L218=), but not the 4-bp promoter deletion. For Duarte variant galactosemia suspicious cases, it is most appropriate to test for the 4-bp GALT promoter deletion to distinguish between the D1 and D2 variants. Hence, integrating biochemical and molecular results can improve the speed and accuracy of a diagnosis by differentiating between pathogenic and non-pathogenic variants as well as cis versus trans combinations.

\section{Galactokinase}

Galactokinase (GALK; EC 2.7.1.6) is the first enzyme in the Leloir pathway (Figure 1). This enzyme belongs to the galactokinase, homoserine kinase, mevalonate kinase, and phosphomevalonate kinase superfamily, and catalyzes the MgATP-dependent phosphorylation of D-galactose to form
Gal-1-P. ${ }^{14}$ It is encoded by GALK1, a gene spanning 8 exons and $7.3 \mathrm{~kb}$ on chromosome $17 \mathrm{q} 25.1 .^{15}$ Deficiency of GALK results in type II galactosemia or galactokinase deficiency (MIM 230200). Several GALK pathogenic variants have been characterized, including insertions, deletions, and single base changes in RBCs. ${ }^{16}$ The most severe phenotype is associated with an insoluble enzyme, while milder phenotypes are characterized by soluble enzyme with impaired catalytic function.

\section{UDP-galactose-4'-epimerase}

UDP-galactose-4'-epimerase (GALE; EC 5.1.3.2) is the third enzyme of the Leloir pathway and catalyzes the reversible conversion of UDP-galactose to UDP-glucose and of UDP- $N$ acetylgalactosamine to UDP- $N$-acetylglucosamine. ${ }^{17}$ GALE is a member of the short-chain dehydrogenase/reductase family of enzymes and is encoded by GALE, located on chromosome 1p36.11. In solution, GALE is a dimer and each subunit contains a binding site with one molecule of the cofactor $\mathrm{NAD}^{+}$. Impaired GALE activity results in type III galactosemia or epimerase deficiency (MIM 230350).

\section{Clinical description of disease}

GALT deficiency (classic galactosemia; type I galactosemia)

Untreated classic galactosemia is often fatal during infancy. Even when identified and treated early, long-term, organspecific complications may occur. Patients typically present in the first two weeks of life with poor feeding, lethargy, vomiting, diarrhea, jaundice, hepatomegaly, and liver failure. Escherichia coli sepsis is often an early complication of the disease. Liver function tests are abnormal as a consequence of the liver involvement. ${ }^{18}$ The withdrawal of lactose (galactose) containing foods from the diet resolves the life-threatening neonatal manifestations; however, even if treated early, patients with classic galactosemia can have complications such as speech dyspraxia, ataxia, and premature ovarian failure in females. ${ }^{18,19}$ A better outcome is associated with the p.S135L variant, common among African American patients, which seems to retain some residual activity in liver and other tissues. ${ }^{6,18}$

Patients with DG variant galactosemia have 15-25\% of normal GALT activity and are usually asymptomatic. ${ }^{12}$ Infants with DG galactosemia on galactose-containing diet (e.g., breast milk or regular formula) have elevated RBC Gal1-P levels and urinary galactitol excretion. ${ }^{20}$ These biochemical abnormalities normalize on a lactose-restricted diet; however, even with an unrestricted diet levels typically normalize during the first year of life. ${ }^{1}$ Despite the biochemical abnormalities, untreated DG galactosemia patients have not demonstrated adverse clinical outcomes in all studies performed to date. ${ }^{1,21,22}$ It is unclear whether this condition is clinically benign or may require lactose restriction, 1,21,22 however, the latest clinical guidelines for the management of patients with galactosemia do not recommend treatment for patients with DG variant galactosemia. $^{23}$ 


\section{GALK deficiency (type I/ galactosemia)}

The only consistent clinical finding in GALK deficiency patients is cataracts. ${ }^{24}$ Other clinical abnormalities including epilepsy, intellectual disability, pseudotumor cerebri, and neurofibromatosis were variably reported in a small percentage of patients (2\%). ${ }^{25}$ In patients identified through newborn screening, dietary galactose restriction initiated within the first two months of life resulted in complete resolution of cataracts. ${ }^{24}$ While blood galactose may reach normal levels in treated patients, galactitol in urine and blood remains elevated. ${ }^{24}$

\section{GALE deficiency (type III galactosemia)}

The clinical consequences of GALE deficiency are variable, ranging from asymptomatic to severe. GALE deficiency comprises three clinical forms: (i) generalized, profound enzyme deficiency in all tissues associated with a specific pathogenic variant, p.V94M; (ii) intermediate, profound deficiency in RBCs and white blood cells and partial deficiency (50\% of normal) in other tissues; and (iii) peripheral, GALE deficiency limited to RBCs and white blood cells. ${ }^{26}$ Patients with generalized GALE deficiency develop clinical symptoms similar to classic galactosemia, including hypotonia, vomiting, jaundice, hepatomegaly, liver dysfunction, and cataracts, which resolve with lactose/galactose restriction. Patients with the peripheral or intermediate forms are typically asymptomatic, and are only identified by an abnormal newborn screen. Several GALE variants associated with the three forms of GALE deficiency have been reported. ${ }^{17,26}$

\section{Newborn screening for galactosemia}

In the United States, all states screen for classic galactosemia (GALT deficiency), the most clinically severe form of the disease. Depending on the screening approach, cases of GALK and GALE deficiencies may also be detected. ${ }^{27}$ The majority of programs use fluorometric Beutler-based methods to semiquantitatively measure GALT activity in DBS. Approximately $30 \%$ of programs measure total galactose (galactose plus Gal-1-P) in DBS either as their primary screening method, or combined with GALT testing. ${ }^{28}$ The presence of elevated galactose sugars together with normal GALT activity suggests a deficiency of either GALK or GALE. ${ }^{26,27}$ The measurement of total galactose alone can lead to falsenegative screening results in galactosemic infants on lactosefree formula and in those receiving total parenteral nutrition. False-positive screening results can be seen in newborns with citrin deficiency, Fanconi-Bickel disease, or liver disease in addition to various medications. ${ }^{29,30}$ DBS GALT activity is stable in refrigerated samples for up to 2 weeks, but with significant loss of activity at higher temperatures or high humidity. ${ }^{31}$ In particular, DBS GALT activity decreases by $>60 \%$ when stored at low humidity and $37^{\circ} \mathrm{C}$ for 32 days, and by $>70 \%$ when stored at high humidity for 1 week. ${ }^{32}$ As a result, this enzyme lability may contribute to false-positive screening results particularly in the summer. Because the
Duarte (D2) allele also reduces enzyme stability, there may be a higher detection rate of DG galactosemia cases in the summer. To increase screening specificity, some programs perform a second-tier DNA test for enzyme or metabolitepositive cases, targeting the most common GALT pathogenic variants. ${ }^{33}$ Additional false-positive results can occur when blood is put into an ethylenediaminetetraacetic acid (EDTA) tube prior to being spotted onto filter paper and in infants with G6PD deficiency. ${ }^{34}$ Transfusion with packed red cells prior to newborn screening can lead to falsely normal GALT enzyme activity. ${ }^{35}$ In these cases it is preferable to proceed with DNA tests if there is a clinical suspicion for galactosemia.

Following an abnormal newborn screen, the diagnosis of classic galactosemia is confirmed by the demonstration of profound deficiency of the GALT enzyme in RBCs and identification of pathogenic variants within the GALT gene by molecular sequencing. States that include total galactose in their screening algorithm can confirm or exclude GALK or GALE deficiency by combining results from the enzyme assay and molecular testing. Historically, DG galactosemia was identified by electrophoresis or isoelectric focusing. ${ }^{12}$ However, with the relative ease and sensitivity of DNA sequencing, these techniques have been replaced with molecular testing.

\section{Incidence}

Classic galactosemia (GALT) attributable to a GG genotype (two pathogenic variants in trans) occurs in 1:53,554 newborns in the United States. ${ }^{36}$ The frequency of DG galactosemia is approximately 10 -fold higher, although the true rate is unknown because of the differences in screening and reporting among states. ${ }^{12}$ The prevalence of GALK deficiency ranges from 1:2,200,000 to $1: 50,000$, with the highest incidence in the Romani population of Bulgaria and Bosnia. ${ }^{24}$ Profound (generalized) GALE deficiency is very rare; epimerase deficiency identified by newborn screening, comprising mostly peripheral/intermediate, is $1: 70,000$ in infants of European descent and 1:6,700 in African Americans. ${ }^{26}$

\section{Mode of inheritance}

All forms of galactosemia are inherited as autosomal-recessive traits.

\section{PREANALYTICAL REQUIREMENTS}

\section{Sample types}

Enzymatic testing is typically performed in RBCs obtained from heparinized whole blood, although other anticoagulants may also be accepted. In patients with classic galactosemia who have received a blood transfusion, donor GALT activity can be detected up to 4 months post transfusion. ${ }^{35}$ In such cases, alternative testing can be used to confirm or exclude a diagnosis of galactosemia, such as blood Gal-1-P, urinary galactitol, or parental testing. GALT mutational analysis can be performed in DNA samples extracted from cultured cells, DBS, or saliva samples when the patient has been transfused with packed RBCs. The buffy coat (thin layer between the 
RBCs and the plasma containing mostly white blood cells and platelets) is also a good source of DNA. As with all DNA testing, DNA from several different sources may be used for GALT mutational analysis as long as the quality and quantity of the extracted DNA is sufficient. The laboratory performing GALT mutational analysis must use validated protocols specific for the sample type and amount of DNA required (ACMG Standards and Guidelines for Clinical Genetics Laboratories, Section G).

\section{Sample volumes}

As a general guideline, 3-5 $\mathrm{ml}$ whole blood (or 1-2 $\mathrm{ml}$ washed $\mathrm{RBC}$ ) is sufficient for measuring GALT, GALK, and GALE activities and Gal-1-P levels, and $2-5 \mathrm{ml}$ of urine for measuring galactitol. Newly developed liquid chromatography-tandem mass spectrometry (LC-MS/MS) based assays are more sensitive and therefore require smaller sample volume. ${ }^{37}$ For mutational analysis of the GALT gene, $1-2 \mathrm{ml}$ whole blood collected in lavender-top (EDTA) tube is preferable. However, DNA extraction yields good results even from heparinized blood. Consult with the testing laboratory for specific sample requirements for unusual situations.

\section{Conditions of sample shipping, handling, and storage}

Whole blood is shipped to the laboratory at room temperature or refrigerated, with special care taken to avoid excessive exposure to heat (which destroys enzyme activity) or cold (which freezes and thereby lyses RBCs). Early studies determined that GALT enzyme activity is stable in whole blood kept refrigerated $\left(4{ }^{\circ} \mathrm{C}\right)$ or at room temperature for up to 2 weeks; however, there are certain pathogenic variants, such as the p.N314D mutant, that produce a more heat-labile protein compared with wild type. ${ }^{31,38}$ The same precautions should be taken when shipping whole blood for Gal-1phosphate testing to avoid loss of Gal-1-P in patients with residual GALT activity. Alternatively, RBCs can be isolated and washed immediately following sample collection and shipped frozen to the testing laboratory. Sample processing instructions should be provided by the testing laboratory, but generally involve centrifuging whole blood to separate RBCs, washing one or more times with normal saline, and freezing the resulting $\mathrm{RBC}$ pellet prior to shipping. $\mathrm{RBC}$ lysates are stable if stored at $-20{ }^{\circ} \mathrm{C}$ or lower. ${ }^{31}$ Urine samples for galactitol testing should be frozen after collection, stored at $-20{ }^{\circ} \mathrm{C}$ or lower, and shipped on dry ice.

\section{METHOD VALIDATION}

As for all laboratory methods used for clinical testing and in agreement with CLIA and the College of American Pathologists (CAP), methods for galactosemia testing should be established and validated by each laboratory. ${ }^{39}$ For enzyme assays, this includes the characterization of optimal buffer conditions, reaction times, and temperatures, as well as relative enzyme and substrate concentrations. Laboratories should verify enzyme stability under different conditions of handling and storage, as well as assay accuracy, sensitivity, analytical measurement range of enzymatic reaction product, and intra- and interday reproducibility. A sample blank containing all assay components except substrate should be used to control for potential matrix interferents. Accuracy can be demonstrated by comparing testing results with an established method, an experienced reference laboratory, or proficiency testing samples. Assay performance characteristics should meet all requirements of CLIA, and should be verified on a regular basis.

\section{Calibration and quantitation}

Appropriate calibration procedures should be performed with each run of sample testing. Criteria for acceptance of calibration and/or of the run should be established. A reportable range for each analyte/enzyme should be determined. If the reportable range lies outside the calibration curve, procedures for verifying this range should be performed at least biannually, according to CLIA/CAP requirements. Calibrators and critical assay reagents, such as substrates and added enzymes, should be qualified/validated prior to their use.

\section{Reference ranges}

Each laboratory should establish reference ranges for enzyme activities and metabolite levels. Affected ranges should be established using positive control samples with a confirmed genotype. An accepted practice is to verify previously established reference ranges, which requires a smaller number of samples (20 samples from an appropriately selected reference population). ${ }^{40}$ Urinary galactitol should be normalized to creatinine and compared with age-specific ranges because of changes in creatinine, and possibly metabolite excretion, with age. GALT, GALK, and GALE enzyme activities and metabolite concentrations in unaffected controls and affected patients are shown in Table 1a-c.

\section{Testing personnel}

Appropriate training and ongoing competency requirements for laboratory personnel performing diagnostic testing for galactosemia must be established and documented. Personnel must satisfy CLIA requirements for high-complexity testing and have, at a minimum, an associate degree in a laboratory science or medical laboratory technology from an accredited institution. Additional specific requirements may apply in some states.

\section{Sample preparation}

\section{SAMPLE ANALYSIS}

The RBC hemolysate for measuring enzyme activity or metabolites is prepared according to individual testing protocols. Enzyme activity is expressed in $\mathrm{U} / \mathrm{g}$ of hemoglobin ( $\mathrm{Hb})$. Measurement of $\mathrm{Hb}$ content $(\mathrm{mg} / \mathrm{ml})$ in hemolysate is performed using standard spectrophotometric protocols. For accurate Gal-1-P quantification, RBCs should immediately be separated from whole blood to avoid loss of Gal-1-P by residual blood GALT enzyme, and washed with cold, normal saline solution. 
Table 1 Enzyme activity and metabolites' concentration in galactosemias

\begin{tabular}{|c|c|c|c|c|}
\hline & $\begin{array}{l}\text { Enzyme activity, } \\
\text { U/Hb (RBCs) }\end{array}$ & $\begin{array}{l}\text { Gal-1-P, }{ }^{a} \mathrm{mg} / \mathrm{dl} \\
\text { (RBCs) }\end{array}$ & $\begin{array}{l}\text { Galactose, }{ }^{a} \mathrm{mg} / \mathrm{dl} \\
\text { (RBCs) }\end{array}$ & $\begin{array}{c}\text { Galactitol, }{ }^{\mathrm{a}} \mathrm{mmol} / \mathrm{mo} \\
\text { Cr (urine) }\end{array}$ \\
\hline Classic galactosemia (G/G) & $<0.20^{\mathrm{b}}$ & $4.3-272^{c}$ & $90-360^{d}$ & $38-335^{c}$ \\
\hline \multirow[t]{2}{*}{ Duarte galactosemia (D/G) } & $3.2-7.9^{f}$ & $14.7-33.8^{f}$ & NR & $54-172^{f}$ \\
\hline & $5.2 \pm 2^{g}$ & & & \\
\hline \multirow[t]{2}{*}{ Unaffected controls } & $15.9-26.4^{f}$ & $<1^{\mathrm{h}}$ & $0-4.3^{i}$ & $29 \pm 23^{h}$ \\
\hline & $27.4 \pm 8^{9}$ & & & \\
\hline \multicolumn{5}{|c|}{ (b) Galactokinase (GALK) deficiency } \\
\hline GALK deficiency & $0-0.15^{i}$ & NR & $160 \pm 94^{i}$ & $11,724 \pm 4,496^{i}$ \\
\hline Unaffected controls & $>1.2^{i}$ & NR & $0-4.3^{i}$ & $29 \pm 23^{h}$ \\
\hline \multicolumn{5}{|c|}{ (c) Galactose-4-epimerase (GALE) deficiency } \\
\hline
\end{tabular}

NR, not reported; RBCs, red blood cells.

${ }^{a}$ The concentrations listed are from treated and untreated patients. See references for specific details. ${ }^{b}$ Ref. $43 .{ }^{c}$ Ref. 19. ${ }^{d}$ Ref. $12 .{ }^{~}$ Ref. $51 .{ }^{f}$ Ref. 1. ${ }^{9}$ Ref. 13. ${ }^{h}$ Ref. 20. iRef. 24. ${ }^{\text {j}}$ Ref. 44. ${ }^{\mathrm{k}}$ Ref. 26.

\section{Analytical methods \\ GALT assays}

There are two widely used methods for measuring GALT activity, one based on UDP-glucose (UDP-glu) consumption and the second on UDP-galactose (UDP-gal) production. In consumption assays, patient hemolysates are incubated with UDP-glu and Gal-1-P, and the residual (unconsumed) UDP-glu is measured by adding $\mathrm{NAD}^{+}$and uridine 5 -diphosphoglucose dehydrogenase to form NADH. NADH absorbs light at $340 \mathrm{~nm}$; the increased absorbance is directly proportional to residual UDP-glu concentration and inversely related to GALT activity. For each sample, a blank is prepared by omitting Gal-1-P from the reaction. Presence of endogenous $\mathrm{NAD}^{+}$can result in false-negative results, especially concerning in newborns and infants in which erythrocytes' NADase is not yet functioning. Preincubation of the sample with NADase should obviate this problem. ${ }^{41}$ Because of this possible confounder, the radioenzymatic assay is considered more specific and sensitive particularly for different tissue types that may vary in endogenous $\mathrm{NAD}^{+}$concentrations.

Radioenzymatic assays directly measure the formation of UDP-gal from a radiolabeled Gal-1-P substrate. ${ }^{42}$ Patient hemolysates are incubated with UDP-glu and ${ }^{14} \mathrm{C}-\mathrm{Gal}-1-\mathrm{P}$ at $37{ }^{\circ} \mathrm{C}$ for $10-30 \mathrm{~min}$, then excess ${ }^{14} \mathrm{C}-\mathrm{Gal}-1-\mathrm{P}$ is separated from ${ }^{14} \mathrm{C}$-UDP-gal product by ion-exchange chromatography. Radioactivity corresponding to each fraction is measured by liquid scintillation counting or other means of radioisotope detection. GALT activity is directly proportional to the amount of ${ }^{14} \mathrm{C}$-UDP-galactose formed. This method is not suitable for high throughput because it involves radioactive material and is labor intensive.
Recently developed ultraperformance LC-MS/MS methods can accurately and reliably measure UDP-galactose production and GALT activity. ${ }^{43,44}$ These methods can be multiplexed and allow the simultaneous determination of enzyme activity for all three galactosemia types with minimal sample volume. This represents a significant improvement for diagnostic testing.

\section{GALK and GALE assays}

GALK and GALE activity are traditionally measured by monitoring production of ${ }^{14} \mathrm{C}-\mathrm{Gal}-1-\mathrm{P}$ and ${ }^{14} \mathrm{C}$-UDP-glucose from ${ }^{14} \mathrm{C}$-galactose and ${ }^{14} \mathrm{C}$-UDP-galactose respectively. Weak cation exchange materials, such as diethylaminoethyl cellulose (DEAE), are used for separation of radiolabeled substrates and products. Recently, LC-MS/MS methods have been developed to measure GALK and GALE activity in RBCs and/or DBS using ${ }^{13} \mathrm{C}$-labeled substrate. ${ }^{43-45}$

\section{Metabolite assays}

Analogous to the GALT consumption assay, a two-step enzymatic reaction followed by spectrophotometric detection of NADH or NADPH can be used to measure Gal-1-P in RBCs. Alkaline phosphatase releases galactose from Gal-1-P, which is then oxidized by galactose dehydrogenase in the presence of $\mathrm{NAD}^{+}$or $\mathrm{NADP}^{+}$to form NADH or NADPH respectively. ${ }^{46} \mathrm{RBC}$ Gal-1-P can also be directly measured by stable isotope dilution gas chromatography/mass spectrometry (GC/MS). ${ }^{47,48}$ GC/MS methods are more sensitive compared with enzymatic methods with a comparable linear range. Galactitol in urine or RBCs can be measured using the same GC/MS methodology. ${ }^{48,49}$ 


\section{Molecular testing}

Targeted testing for common pathogenic variants in GALT is frequently performed; however, if this testing is inconsistent with biochemical findings, GALT gene sequencing or genetargeted deletion/duplication testing is appropriate and useful in the interpretation of equivocal biochemical testing results. For example, since heterozygotes for classic galactosemia $(G / g)$, Duarte variant homozygotes $(D 2 / D 2)$, or LA variant galactosemia $(D 1 / G)$ all have approximately $50 \%$ of the normal enzyme activity, molecular testing can distinguish between these three different allele combinations. Since the D1 variant is not associated with decreased GALT activity, this allele should be reported as benign when detected. ${ }^{12}$ Molecular testing of the proband, along with follow-up parental testing, identifies the GALT alleles present in the family and allows for a more accurate risk assessment of a couple having a child with classic galactosemia than biochemical testing alone.

Full-gene sequencing followed by gene-targeted deletion/ duplication testing, rather than targeted mutation testing, is typically performed for GALE and GALK. ${ }^{26}$

\section{Quality control}

A positive (affected) and negative (normal) control should be included in each batch of patient samples for all enzyme assays. ${ }^{39}$ Given the relatively high volume of RBCs needed, positive (low enzyme activity) and negative (normal enzyme activity) controls are usually prepared using pools of RBCs. Positive controls can be heat inactivated prior to use. For metabolite quantification, at least two control concentrations should be included in each analytical run. Acceptable ranges for quality controls (QCs) should be established prior to their use for clinical testing with at least 20 (ideally 30) data points obtained in different runs on different days. Criteria for acceptance or rejection of quality controls, and remedial procedures for failed QCs, should be defined in the laboratory protocol. QC material should be stored in aliquots at $-20{ }^{\circ} \mathrm{C}$ or lower. Periodic review of QCs and QC activity should be performed by the laboratory supervisor and/or director.

\section{Proficiency testing}

Proficiency testing for galactosemia enzymes and metabolites should be carried out at least twice per year. ${ }^{39}$ Currently, there are no external proficiency testing programs for galactosemia (enzymes or metabolites). The Clinical and Laboratory Standards Institute and the CAP suggest alternative proficiency testing approaches such as interlaboratory sample exchange or internal testing (reanalyzing previously tested material in a blind fashion).

\section{TEST INTERPRETATION AND REPORTING Interpretation \\ Enzyme activity}

Reduced GALT activity should be interpreted in the context of historical and concurrent unaffected control samples. A reduced GALT activity of $\leq 3 \%$ of normal is usually diagnostic of classic galactosemia (Table 1a). The GALT activity of DG $(D 2 / G)$ galactosemia patients is approximately $25 \%$ of controls, while the activity of heterozygotes for classic galactosemia $(G / g)$, Duarte variant homozygotes $(D 2 / D 2)$, or LA variant galactosemia $(D 1 / G)$ is approximately $50 \%$ of the normal activity. Heterozygotes for the Duarte (D2) allele usually have $75 \%$ of normal enzyme activity and may not always be distinguishable from normal controls or carriers based solely on enzyme activity. Carriers of the LA variant (D1) have normal to supranormal enzyme activity. Molecular testing, targeted or full-gene sequencing and gene-targeted deletion/duplication testing, is useful in confirming the diagnosis, and should be correlated with biochemical results whenever possible. GALK activity in galactokinase-deficient patients varies from zero to approximately $10 \%$ of normal ${ }^{24}$ (Table 1b). UDP-galactose- 4 '-epimerase (GALE) deficiency can be limited to erythrocytes (peripheral form), the cells usually tested, or also affect the liver and other tissues (generalized form) (Table 1c). Given the different presentations DNA sequencing analysis of the GALE gene may help to determine the clinical significance of deficient GALE activity.

\section{Metabolites}

Untreated patients with classic galactosemia have markedly elevated RBC Gal-1-P concentrations (Table 1) that decrease rapidly upon elimination of galactose from the diet. Gal-1-P is routinely used to monitor response to therapy and dietary compliance. It usually takes $2-3$ months for Gal-1-P to fall within the therapeutic range $(2-4 \mathrm{mg} / \mathrm{dl}) .{ }^{50}$ Patients with DG galactosemia often show elevated Gal-1-P in the first weeks of life, even up to $30-40 \mathrm{mg} / \mathrm{dl}$, after which concentrations normalize without dietary intervention. The combination of endogenous production ${ }^{51}$ and dietary intake of galactose with the reduced enzyme activity may underlie this transient elevation of Gal-1-P. Galactitol accumulates in tissues and is excreted in the urine of patients with different forms of galactosemia. Baseline values of galactitol differ greatly between patients and may be affected by age and body mass. Early studies demonstrated that, although both urinary galactitol and Gal-1-P are greatly increased in patients with classic galactosemia, urinary galactitol may be a better marker of dietary compliance; ${ }^{20,48,49,52}$ however, the clinical utility of galactitol for managing patients with classic galactosemia is still considered limited. ${ }^{23}$

\section{Reporting}

Written reports should follow appropriate guidelines (ACMG Standards and Guidelines for Clinical Genetics Laboratories, Section C 2.4, 2.4.1, and 2.4.2 and CLIA) and include patient and specimen information as given, enzyme activity or metabolite concentrations, units of measure, a reference range, and an interpretation. For molecular test reports, variants identified by common mutation panels or by fullgene sequencing and gene-targeted deletion/duplication analysis should be interpreted according to ACMG 
guidelines. $^{53}$ Recommendations for follow-up, including referral to a metabolic specialist, should also be included when appropriate.

\section{ACKNOWLEDGMENTS}

The authors acknowledge Tina Cowan and Anna Scott for their critical review of this document.

\section{DISCLOSURE}

M.P. is associated with a biochemical genetics laboratory that performs diagnostic testing for galactosemia. B.C. directed a lab that ran the tests discussed in this Technical Standard and Guideline during the time this document was being developed. However, since 2014, he has not been involved with clinical laboratory testing for the diagnosis of galactosemia. C.Y. declares no conflict of interest.

\section{REFERENCES}

1. Ficicioglu C, Thomas N, Yager C, et al. Duarte (DG) galactosemia: a pilot study of biochemical and neurodevelopmental assessment in children detected by newborn screening. Mol Genet Metab. 2008;95:206-212.

2. Brenner C. Hint, Fhit, and GalT: function, structure, evolution, and mechanism of three branches of the histidine triad superfamily of nucleotide hydrolases and transferases. Biochemistry. 2002;41: 9003-9014.

3. Frey PA. The Leloir pathway: a mechanistic imperative for three enzymes to change the stereochemical configuration of a single carbon in galactose. FASEB J. 1996;10:461-470.

4. Calderon FR, Phansalkar AR, Crockett DK, Miller M, Mao R. Mutation database for the galactose-1-phosphate uridyltransferase (GALT) gene. Hum Mutat. 2007;28:939-943.

5. Tyfield L, Reichardt J, Fridovich-Keil J, et al. Classical galactosemia and mutations at the galactose-1-phosphate uridyl transferase (GALT) gene. Hum Mutat. 1999;13:417-430.

6. Lai K, Elsas LJ. Structure-function analyses of a common mutation in blacks with transferase-deficiency galactosemia. Mol Genet Metab. 2001;74:264-272

7. Goldstein N, Cohen Y, Pode-Shakked B, et al. The GALT rush: high carrier frequency of an unusual deletion mutation of the GALT gene in the Ashkenazi population. Mol Genet Metab. 2011;102:157-160.

8. Coffee B, Hjelm LN, DeLorenzo A, Courtney EM, Yu C, Muralidharan K. Characterization of an unusual deletion of the galactose-1-phosphate uridyl transferase (GALT) gene. Genet Med. 2006;8:635-640.

9. Crushell E, Chukwu J, Mayne P, Blatny J, Treacy EP. Negative screening tests in classical galactosaemia caused by $\mathbf{S 1 3 5 L}$ homozygosity. J Inherit Metab Dis. 2009;32:412-415.

10. Elsas LJ, Langley S, Steele E, et al. Galactosemia: a strategy to identify new biochemical phenotypes and molecular genotypes. Am J Hum Genet. 1995;56:630-639.

11. Carney $A E$, Sanders RD, Garza $K R$, et al. Origins, distribution and expression of the Duarte-2 (D2) allele of galactose-1-phosphate uridylyltransferase. Hum Mol Genet. 2009;18:1624-1632.

12. Fridovich-Keil JL, Gambello MJ, Singh RH, Sharer JD. Duarte variant galactosemia. In: Pagon RA, Adam MP, Ardinger $\mathrm{HH}$, et al., (eds). GeneReviews. University of Washington: Seattle, WA, 1993.

13. Langley SD, Lai K, Dembure PP, Hjelm LN, Elsas LJ. Molecular basis for Duarte and Los Angeles variant galactosemia. Am J Hum Genet. 1997;60:366-372.

14. Thoden JB, Timson DJ, Reece RJ, Holden HM. Molecular structure of human galactokinase: implications for type II galactosemia. J Biol Chem. 2005;280:9662-9670.

15. Bergsma DJ, Ai $Y$, Skach WR, et al. Fine structure of the human galactokinase GALK1 gene. Genome Res. 1996;6:980-985.

16. Timson DJ, Reece RJ. Functional analysis of disease-causing mutations in human galactokinase. Eur J Biochem. 2003;270:1767-1774.

17. Timson DJ. The structural and molecular biology of type III galactosemia. IUBMB Life. 2006;58:83-89.
18. Berry GT. Classic galactosemia and clinical variant galactosemia. In: Pagon RA, Adam MP, Ardinger HH, et al. (eds). GeneReviews. University of Washington: Seattle, WA, 1993.

19. Webb AL, Singh RH, Kennedy MJ, Elsas LJ. Verbal dyspraxia and galactosemia. Pediatr Res. 2003;53:396-402.

20. Ficicioglu C, Hussa C, Gallagher PR, Thomas N, Yager C. Monitoring of biochemical status in children with Duarte galactosemia: utility of galactose, galactitol, galactonate, and galactose 1-phosphate. Clin Chem. 2010;56:1177-1182.

21. Lynch ME, Potter NL, Coles CD, Fridovich-Keil JL. Developmental outcomes of school-age children with Duarte galactosemia: a pilot study. JIMD Rep. 2015;19:75-84.

22. Powell KK, Van Naarden Braun K, Singh RH, Shapira SK, Olney RS, Yeargin-Allsopp M. Long-term speech and language developmental issues among children with Duarte galactosemia. Genet Med. 2009;11: 874-879.

23. Welling L, Bernstein LE, Berry GT, et al. International clinical guideline for the management of classical galactosemia: diagnosis, treatment, and follow-up. J Inherit Metab Dis. 2016;40:171-176.

24. Hennermann JB, Schadewaldt P, Vetter B, Shin YS, Monch E, Klein J. Features and outcome of galactokinase deficiency in children diagnosed by newborn screening. J Inherit Metab Dis. 2011;34:399-407.

25. Bosch AM, Bakker HD, van Gennip AH, van Kempen JV, Wanders RJ, Wijburg FA. Clinical features of galactokinase deficiency: a review of the literature. J Inherit Metab Dis. 2002;25:629-634.

26. Fridovich-Keil J, Bean L, He M, Schroer R. Epimerase deficiency galactosemia. In: Pagon RA, Adam MP, Ardinger $\mathrm{HH}$, et al. (eds). GeneReviews. University of Washington: Seattle, WA, 1993.

27. Kaye $\mathrm{Cl}$, Committee on Genetics, Accurso F, et al. Newborn screening fact sheets. Pediatrics. 2006;118:e934-963.

28. National Newborn Screening Status Report. http://genes-r-us.uthscsa. edu/sites/genes-r-us/files/nbsdisorders.pdf. Accessed 10 May 2017.

29. Kobayashi K, Saheki T, Song YZ. Citrin deficiency. In: Pagon RA, Adam MP, Ardinger $\mathrm{HH}$, et al. (eds). GeneReviews. University of Washington: Seattle, WA, 1993.

30. Peduto A, Spada M, Alluto A, La Dolcetta M, Ponzone A, Santer R. A novel mutation in the GLUT2 gene in a patient with Fanconi-Bickel syndrome detected by neonatal screening for galactosaemia. J Inherit Metab Dis. 2004;27:279-280.

31. Beutler E, Baluda MC. Improved method for measuring galactose-Iphosphate uridyl transferase activity of erythrocytes. Clin Chim Acta. 1966;13:369-379

32. Adam BW, Hall EM, Sternberg M, et al. The stability of markers in driedblood spots for recommended newborn screening disorders in the United States. Clin Biochem. 2011:44:1445-1450.

33. Sartippour MR, Doroudian R, Frampton G, et al. Identification of galactose-1-phosphate uridyl transferase gene common mutations in dried blood spots. Clin Chim Acta. 2014;436:298-302.

34. Fujimoto A, Okano Y, Miyagi T, Isshiki G, Oura T. Quantitative Beutler test for newborn mass screening of galactosemia using a fluorometric microplate reader. Clin Chem. 2000;46:806-810.

35. Sokol RJ, McCabe ER, Kotzer AM, Langendoerfer SI. Pitfalls in diagnosing galactosemia: false negative newborn screening following red blood cell transfusion. J Pediatr Gastroenterol Nutr. 1989;8:266-268.

36. Therrell Jr. BL, , Lloyd-Puryear MA, Camp KM, Mann MY. Inborn errors of metabolism identified via newborn screening: ten-year incidence data and costs of nutritional interventions for research agenda planning. Mol Genet Metab. 2014;113:14-26.

37. Li Y, Ptolemy AS, Harmonay L, Kellogg M, Berry GT. Quantification of galactose-1-phosphate uridyltransferase enzyme activity by liquid chromatography-tandem mass spectrometry. Clin Chem. 2010;56:772-780.

38. Lai K, Langley SD, Dembure PP, Hjelm LN, Elsas L 2nd. Duarte allele impairs biostability of galactose-1-phosphate uridyltransferase in human lymphoblasts. Hum Mutat. 1998;11:28-38.

39. Centers for Disease Control and Prevention. Good laboratory practices for biochemical genetic testing and newborn screening for inherited metabolic disorders. MMWR Recomm Rep. 2012;61:1-44.

40. Clinical and Laboratory Standards Institute. Defining, establishing, and verifying reference intervals in the clinical laboratory. Approved Guideline - Third Edition. CLSI Document EP28-A3C. Wayne, PA, 2010.

41. De Bruyn CH, Raymakers C, Wensing A, Oei TL. Galactose-1-phosphate uridyltransferase activities in erythrocytes from a patient with galactosemia: discrepancy between two methods. Clin Chim Acta. 1977;78: 145-150. 
42. Xu YK, Kaufman FR, Donnell GN, Ng WG. Radiochemical assay of minute quantities of galactose-1-phosphate uridyltransferase activity in erythrocytes and leukocytes of galactosemia patients. Clin Chim Acta. 1995;235:125-136.

43. Ko DH, Jun SH, Park HD, et al. Multiplex enzyme assay for galactosemia using ultraperformance liquid chromatography-tandem mass spectrometry. Clin Chem. 2010;56:764-771.

44. Li Y, Ptolemy AS, Harmonay L, Kellogg M, Berry GT. Ultra fast and sensitive liquid chromatography tandem mass spectrometry based assay for galactose-1-phosphate uridylyltransferase and galactokinase deficiencies. Mol Genet Metab. 2011;102:33-40.

45. Chen J, Meyers GA, Bennett MJ. An interference-free two-step enzyme assay with UPLC-tandem mass spectrometric product measurement for the clinical diagnosis of uridine diphosphate galactose-4-epimerase deficiency. J Chromatogr B Analyt Technol Biomed Life Sci. 2014;959:5-9.

46. Pesce MA, Bodourian SH, Nicholson JF. A new microfluorometric method for the measurement of galactose-1-phosphate in erythrocytes. Clin Chim Acta. 1982;118:177-189.

47. Chen J, Yager C, Reynolds R, Palmieri M, Segal S. Erythrocyte galactose 1phosphate quantified by isotope-dilution gas chromatography-mass spectrometry. Clin Chem. 2002;48:604-612.
48. Schadewaldt P, Kamalanathan L, Hammen HW, Wendel U. Stableisotope dilution analysis of galactose metabolites in human erythrocytes. Rapid Commun Mass Spectrom. 2003;17:2833-2838.

49. Ficicioglu C, Yager C, Segal S. Galactitol and galactonate in red blood cells of children with the Duarte/galactosemia genotype. Mol Genet Metab. 2005;84:152-159.

50. Berry GT, Nissim I, Lin Z, Mazur AT, Gibson JB, Segal S. Endogenous synthesis of galactose in normal men and patients with hereditary galactosaemia. Lancet. 1995;346:1073-1074.

51. Berry GT, Nissim I, Gibson JB, et al. Quantitative assessment of whole body galactose metabolism in galactosemic patients. Eur J Pediatr. 1997;156(suppl 1):S43-49.

52. Palmieri M, Mazur A, Berry GT, et al. Urine and plasma galactitol in patients with galactose-1-phosphate uridyltransferase deficiency galactosemia. Metabolism. 1999;48:1294-1302.

53. Richards S, Aziz N, Bale S, et al. Standards and guidelines for the interpretation of sequence variants: a joint consensus recommendation of the American College of Medical Genetics and Genomics and the Association for Molecular Pathology. Genet Med. 2015; 17:405-424. 